\title{
A Study on Classification Using Machine Learning for Dementia Evaluation
}

\author{
Kanta Umemura*, Hiroharu Kawanaka*, Yulia Hicks ${ }^{\dagger}$, Rossitza Setchi ${ }^{\dagger}$, Daisuke Takamatsu ${ }^{\ddagger}$, Shinji Tsuruoka * \\ ${ }^{*}$ Graduate School of Engineering, Mie University, Kurima-machiya, Tsu, Mie 514-8507, Japan \\ Email: \{419m209@m,kawanaka@elec\}.mie-u.ac.jp \\ $\dagger$ Cardiff University, Cardiff, UK \\ $\ddagger$ Sofical Welfare Corporation, Taiyo-no-Sato, Mie, Japan
}

\begin{abstract}
Recently, the number of dementia patients has been increasing due to the aging society. In Japan, a paper-based examination is the main-stream to measure the cognitive function of a subject, but these paper-based tests give much burden to not only patients but also evaluators like facility and medical staff. Therefore, it is necessary to develop a system that can automatically judge the degree of dementia progression, not to burden the doctor. Also, it is required to add play ability not to be a burden on the elderly. From this point of view, the authors developed a recreation game like a puzzle game. This system is easy to play for elderly people and is not a burden. Also, the question-answer is clear, so it is suitable for automatic judgment. We use the obtained features during recreation game to diagnose the degree of dementia progression. We committed the capability of machine learning techniques. Finally, we discussed that the collected features are sufficient to diagnose the degree of dementia progression.
\end{abstract}

Index Terms-Dementia, Recreation Game, Automatic Judgment, Hand Motor Function, Machine Learning

\section{INTRODUCTION}

Japan is one of the fastest aging countries in the world. As a result of aging, the elderly population with dementia increases gradually. The increase in dementia patients is a significant social problem. This situation shows that prevention and improvement of dementia is an important issue.

Mini-Mental State Examination (MMSE), Clock Drawing Test (CDT) and Montreal Cognitive Assessment (MoCA) are usually used to evaluate a patient's cognitive functions. Usually, these methods evaluate a patient's cognitive function(s) and finally judge their dementia progression. However, these approaches have some problems as follows. First, a patient becomes very nervous about the evaluation test. Second, these evaluation tests give much burden to the doctors/nurses for follow-up [1]. From this point of view, it is necessary to develop a system that is able to automatically judge the degree of dementia progression without much burden to medical/facility staff. Also, play ability without a burden on subjects is also required for these systems.

On the other hand, Emi et al. showed that playing a musical instrument and cooking are effective in recovering brain function [2], [3] because both hands are usually used while playing musical instruments and cooking. From this point of view, many nursing homes use exercise using both hands and brain function. Also, Tachibana evaluated a subject's cognitive function using the pegboard test. Figure 1 shows the image of the pegboard test. The pegboard test measures how many pins the subject can move in time to assess the hand dexterity. This literature discussed the relationship between the pegboard test score and cognitive function [4], [5]. Therefore, we also focus on hand dexterity, such as the speed of moving hands.

In this paper, the authors aim to develop an automatic system to evaluate dementia progression quantitatively. We focus on the relationship between cognitive function and hand motor function. We are now developing a new recreational system to evaluate the hand motor and cognitive function of elderly people.

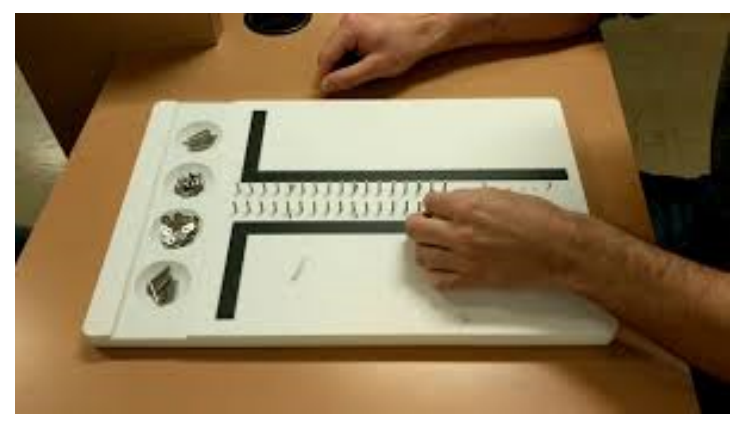

Fig. 1. Pegboard Test

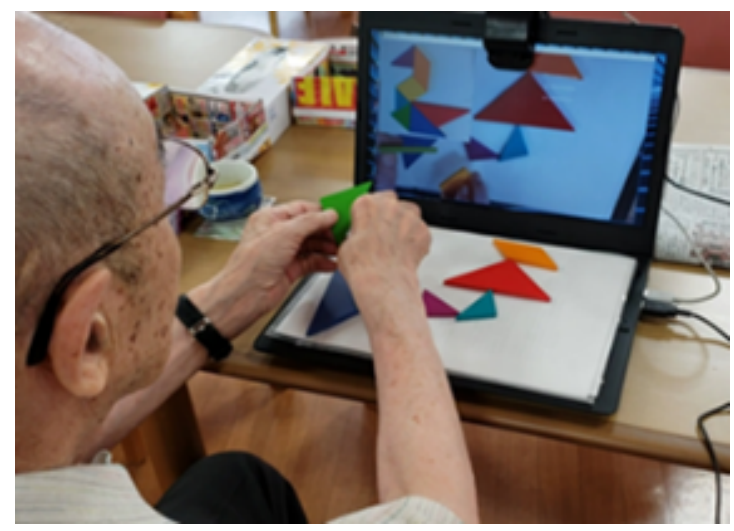

Fig. 2. Recreation Game 


\section{DEVELOPED SYSTEM FOR RECREATION}

\section{A. Simple Game Using Tangram}

Figure 2 shows the elderly person are playing the recreational game. In the developed system, the authors use a puzzle game like a tangram. Tangram is a kind of puzzle game with triangle and rectangle pieces. This system is easy to play for subjects i.e., elderly people, and does not give much burden to them and also, subjects can use their hands and figures naturally.

\section{B. Outline of Developed System}

During the game, the system measures movements of a user's hands in real-time. Figure 3 shows an example of obtained data by the developed system. In Fig. 3, each column means coordinates $(x, y, z)$, time, and velocity of the user's hand, respectively. Figure 4 shows the coordinate system for our system. As you can see, the movement of the user's hand can be measured in real-time with the system, and the obtained data can be analyzed to evaluate the user's hand motor function.

In this paper, the feature descriptors shown in Table I were calculated from the obtained data. These descriptors are defined by equations (2), (3), and (4). From these feature values, we can acquire information about the movement of both hands and hand dexterity.

TABLE I

The Feature Value of Hand Motor Function

\begin{tabular}{c|l|c}
\hline & Feature Value & Unit \\
\hline$(0)$ & Dominant Hand & L or R \\
$(1)$ & Percentage of Both Hands & $\%$ \\
$(2)$ & Percentage of Only Dominant Hand & $\%$ \\
$(3)$ & Percentage of Only No-Dominant Hand & $\mathrm{cm}$ \\
$(4)$ & The Average Speed of Dominant Hand & $\mathrm{cm}$ \\
\hline
\end{tabular}

\begin{tabular}{|l|r|r|r|r|r|}
\hline \multicolumn{1}{|c|}{ A } & \multicolumn{1}{|c}{ A } & B & \multicolumn{1}{c}{ C } & D & \multicolumn{1}{c|}{ E } \\
\cline { 2 - 7 } 1 & 41.4239 & 7.206902 & 26.5918 & 1.075 & 165.984 \\
\hline 2 & 26.30521 & -7.72314 & 73.75183 & 1.168 & 556.1909 \\
\hline 3 & 26.92762 & -7.8913 & 73.73245 & 1.179 & 58.63767 \\
\hline 4 & 27.68273 & -7.93451 & 73.83725 & 1.188 & 84.84246 \\
\hline 5 & 27.5699 & -7.84102 & 74.26281 & 1.199 & 40.91629 \\
\hline 6 & 27.58493 & -7.55998 & 74.79543 & 1.208 & 65.93485 \\
\hline 7 & 27.84359 & -7.17458 & 75.13906 & 1.218 & 57.74979 \\
\hline 8 & 28.25952 & -6.77379 & 75.31226 & 1.229 & 54.8206 \\
\hline 9 & 28.70437 & -6.48926 & 75.83572 & 1.239 & 74.35487 \\
\hline 10 & 29.0735 & -6.24163 & 76.1963 & 1.251 & 47.69539 \\
\hline 11 & 30.0721 & -5.5733 & 75.97714 & 1.258 & 174.4912 \\
\hline 12 & 30.49419 & -5.10527 & 75.88556 & 1.269 & 57.89672 \\
\hline
\end{tabular}

Fig. 3. Collected Data by our System

\section{Methodology}

\section{A. Data Collection}

This research project is a collaboration project with the social welfare corporation, Taiyo-no-Sato in Matsusaka city,

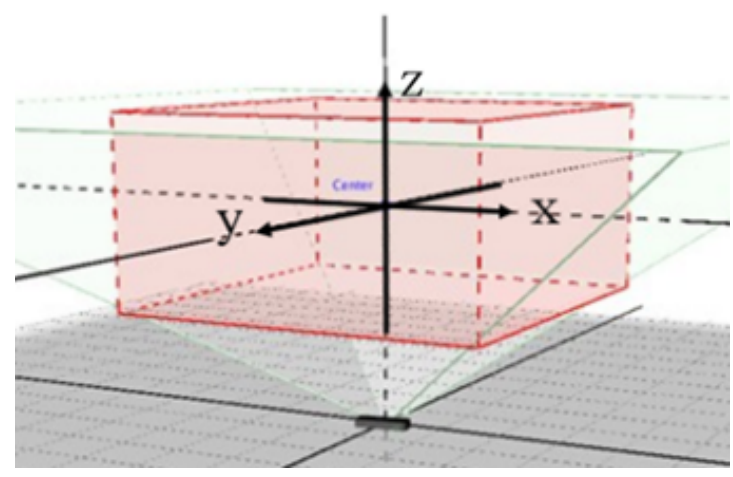

(a) Coordinate System

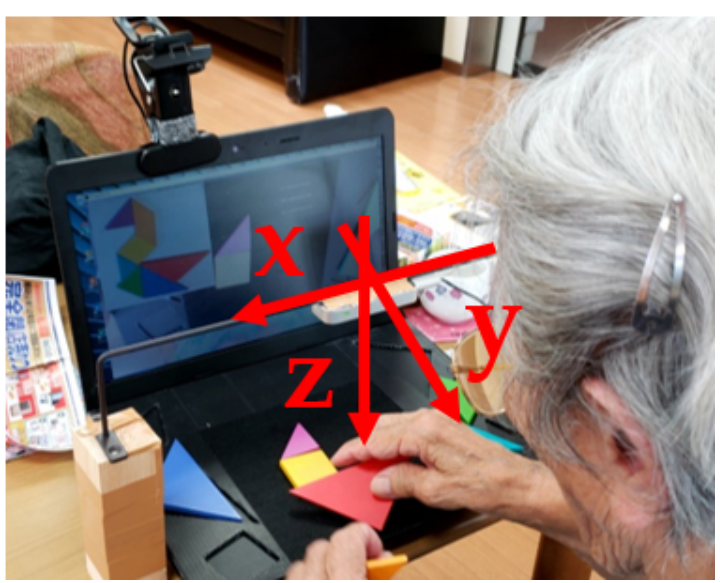

(b) Relationship between Coordinate System and Hand Positions

Fig. 4. Coordinate System Used for Developed System

Mie Pref. In this experiment, the authors focused on subjects whose age was from 78 to 96 years old. The authors collected the data from 144 subjects. This paper also targeted elderly people whose levels of motor function was from low-level to high-level. In this paper, "low-level" means that a subject's motor function is almost no problem for his/her daily life, and a subject who is regarded as "high-level" cannot walk alone. In this paper, all of subjects do not have a problem of hand motor function.

\section{B. Experimental Materials}

In this paper, the authors focused on the relationship between hand motor function and cognitive function. The authors also measured (1) long-term memory ranked from 0 to 3 points, and (2) short-term memory ranked from 0 to 8 points as cognitive functions.In the experiment, three questions are usually used to measure long-term memory as follows;

1) What year is it today

2) What month is today

3) What is the date today

Short-term memory can be evaluated how many images the subject can memorize in time. By using the scores of the above two tests, we classified the level of short-term memory of the subject into three groups shown (Table. III). 


$$
\text { Percentage of moving both hands }(\%)=\frac{\text { Movement time of both hand }(s)}{\text { Answer time }(s)}
$$

Percentage of moving only dominant hand $(\%)=\frac{\text { Movement time of both hand }(s)}{\text { Answer time }(s)}$

$$
\text { Percentage of moving only no-dominant hand }(\%)=\frac{\text { Movement time of both hand }(s)}{\text { Answer time }(s)}
$$

TABLE II

The Collected Data of Cognitive Function

\begin{tabular}{l|ll}
\hline Cognitive Test & Feature Value & \\
\hline \hline Long-term Memory & The Number of Correct Answers & $(0$ to 3) \\
Short-term Memory & The Number of Memorized Images & $(0$ to 8$)$ \\
Sum of Two Tests & Total Score & (0 to 11) \\
\hline
\end{tabular}

TABLE III

The Collected Data of Cognitive Function

\begin{tabular}{c|c|c}
\hline Dementia Progression & Score & \# of People \\
\hline Severe Progression & 0 to 3 points & 30 \\
Mild Progression & 4 to 6 Points & 74 \\
Healthy Progression & 7 to 11 Points & 40 \\
\hline
\end{tabular}

\section{Classification Using SVM}

In this experiment, the authors experimented whether we can classify into three groups (shown in Table III) using Support Vector Machine. Since the number of collected data is insufficient, we added Gaussian noise to expand the amount of data. As a testing method, we use the cross-validation method. We calculated the average accuracy of the cross-validation experiments.

\section{RESUlts AND Discussion}

In this experiment, the authors focus on the relationship between cognitive and recreation game data. Also, the authors experimented whether we can classify into three groups using obtained data and machine learning. As a result of the experiment, we obtained $82 \%$ of classification accuracy. We believe that the obtained accuracy was enough for practical use, i.e., daily recreation. Also, we confirmed that the obtained features were significant for an estimation of the degree of dementia progression. From this point of view, we can conclude that there is a high relationship between hand motor function and cognitive function.

However, we employed three-class classification, and it will not be enough to introduce this system to a nursing home because more detailed evaluation level is required for practical use. Also, the number of feature values is not enough to classify more than four groups. Therefore, it is necessary to collect more data and search for significant features continuously. On the other hand, almost subjects (elderly persons in the care house) did not play this recreation game reluctantly. From this point of view, we can say that the developed system satisfies in terms of play-ability.

\section{CONCLUSION}

In this paper, the authors aimed to develop a new system to solve the problems of the dementia evaluation test. The authors found that there was a deep relationship between memory functions and the obtained data during the developed recreation game. Also, we could confirm that the collected features were sufficient to classify the degree of dementia progression.

However, the number of collected data was not enough. We could not show the above relation at the moment. More experimental materials will be required for advanced discussions. As future works of this research project, the authors will continue to collect experimental materials. Also, we have to look for other features to classify more than four class classification and improve the classification accuracy.

\section{REFERENCES}

[1] Hisao Shiizuka, A Survey of Dementia Assessment Scales and Its Related Problems, Research reports of the Kogakuin University, Vol. 111, pp.137 - 144, 2011

[2] Emi Ito, et al., Effect of leisure activities on cognitive functions, Journal of Human Environmental Studies, Vol. 1, pp.15 - 20, 2004

[3] Machiko Yamashita, et al., Measurements of brain activity when cooking using a near infrared measurement device: optical topography system, Toho Journal, Vol. 17, No. 2, pp.125 - 129, 2006

[4] Son Bo-Young, Effect of task-oriented activities on hand functions, cognitive functions and self-expression of elderly patients with dementia, J Phys Ther Sci., Aug;29(8), pp.1357 - 1362, 2017

[5] Hiro Tachibana, Finger Dexterity and Prefrontal Cortex Activity in Pegboard Performance, Toho Journal, Vol. 47, No. 2, pp.109 - 117, 2018 